\title{
Influence of orientation-dependent grain boundary oxidation on fatigue cracking behaviour in an advanced $\mathrm{Ni}$-based superalloy
}

\author{
R. Jiang*, N. Gao, P. A. S. Reed \\ Materials Research Group, Engineering and the Environment, University of Southampton, \\ Highfield, Southampton, SO17 1BJ, UK
}

Tel: (+44) 023 80599450; Fax: (+44) 0238059 3016; Email: Rong.Jiang@soton.ac.uk

\begin{abstract}
Fatigue tests have been conducted on an advanced disc Ni-based superalloy (Low Solvus, High Refractory (LSHR) alloy) at $650{ }^{\circ} \mathrm{C}$ in air under three-point bend loading to investigate the role of orientation-dependent grain boundary oxidation in crack initiation and early propagation. It is found that crack initiation occurs mainly from bulged grain boundary oxides, and cracks then predominantly propagate along the oxidised grain boundaries. These bulged oxides are extremely enriched in $\mathrm{Co}$ and preferentially form at the boundaries between high and low Schmid factor grains which are inclined normal to the applied tensile stress direction. Meanwhile, relatively flat/thin Ni/Ti/Al-rich oxide complexes also form at other grain boundaries, but they appear to be much less detrimental in fatigue crack initiation and propagation compared with the grain boundary bulged Co-rich oxide complexes.
\end{abstract}

Keywords: Ni-based superalloy; fatigue; grain boundary oxidation; Schmid factor; Co-rich oxide

\section{Introduction:}

Powder metallurgy Ni-based superalloys are widely used for aeroengine turbine disc application due to their exceptional strength properties at elevated temperatures, good fatigue and creep performance as well as excellent corrosion and oxidation resistance. However, oxidation enhanced fatigue crack initiation and propagation at elevated temperatures in air has been commonly observed in turbine disc superalloys under dwell fatigue testing conditions [1-10]. Generally, it is considered that enhanced crack initiation is mainly caused by oxide cracking and/or slip band cracking due to reduced slip reversibility resulting from the absorption of oxygen at the slip band/matrix interface [11]. It is also reported that the dissolution of the grain boundary (GB) carbides due to oxide formation promoted intergranular crack initiation and thereby was associated with a shorter fatigue lifetime in the disc superalloys [12]. The enhanced crack propagation associated with intergranular fracture 
is usually ascribed to decohesion/reduction in cohesion strength of GBs ahead of the crack tip due to dynamic embrittlement [13-15] or GB oxide/matrix-oxide interface cracking caused by stress assisted grain boundary oxidation (SAGBO) [5, 16-18].

Extensive studies on SAGBO have shown that the most common oxides observed in the disc alloys are oxides of $\mathrm{Ni}, \mathrm{Cr}, \mathrm{Nb}$, Ti and $\mathrm{Al}[3,16,17,19-21]$, as well as Co-rich oxides in those superalloys with relatively high Co content such as RR1000 [18]. These oxide complexes usually possess a layered structure which consists of a thermodynamically unstable central layer of $\mathrm{NiO} / \mathrm{CoO}$, a thermodynamically stable intermediate layer of $\mathrm{Cr}_{2} \mathrm{O}_{3}$ and a marginal layer of $\mathrm{Al}_{2} \mathrm{O}_{3} / \mathrm{TiO}_{2}[3,16,18]$. Moreover, study of isothermal oxidation and/or cyclic oxidation in disc alloys at elevated temperatures also shows similar oxide layers at surfaces which usually consist of an external $\mathrm{Cr}_{2} \mathrm{O}_{3}$ layer and an internal $\mathrm{Al}_{2} \mathrm{O}_{3}$ layer $[12$, $22]$. Formation of such oxides at/or ahead of the crack tip and/or at specimen surface mostly depends on the composition of the investigated disc alloys and environmental conditions such as $\mathrm{O}_{2}$ partial pressure. As suggested by Molins et al. [3], the enhanced fatigue crack propagation in Inconel 718 results from the formation of intergranular Ni oxide nuclei rather than protective chromia at the crack tip. By increasing the content of $\mathrm{Cr}$ in the matrix or reducing the $\mathrm{O}_{2}$ partial pressure, it is possible to promote formation of chromia and thereby suppress the detrimental effect of oxidation. The formation of oxides is also related to the accumulated strain during cyclic loading which is closely related to grain orientation as it may influence the local slip system activity and slip transmission across a GB [23]. The higher accumulated strain is usually associated with a greater number of dislocations and vacancies produced during the deformation process, which can facilitate the diffusion of the oxide-forming elements and accelerate oxide formation.

Although the role of SAGBO in oxidation enhanced fatigue crack initiation and propagation in turbine disc superalloys has been extensively examined and recognized, most of these studies were based on the post-test observation of the fracture surface along with a focus on the thickness of the oxide layer. The dependence of the formation and evolution of GB oxides on grain orientation (which is associated with deformation process) and their role in subsequent crack initiation and propagation are not clear. In addition, the detrimental oxides formed under cyclic loading are diverse due to the complex compositions of turbine disc superalloys and studies regarding the nature of GB oxides are limited, especially in the more recently developed turbine disc alloys, such as the Low Solvus, High Refractory (LSHR) alloy developed by NASA. The LSHR alloy combines the low solvus of ME3 brought about 
by the high Co content and the high refractory element content of Alloy 10 [24]. The comparatively high Co content lowers the solvus temperature to allow more flexible solution heat treatment and increases the strength by increasing mechanical twining during deformation at elevated temperatures due to the low stacking fault energy $[25,26]$. In this study, fatigue tests were conducted on the LSHR alloy at $650{ }^{\circ} \mathrm{C}$, with a particular focus on studying the influence of the orientation-dependent GB oxide formation on fatigue crack initiation and propagation processes

\section{Materials and experimental procedures}

Supersolvus heat treated LSHR alloy with an average grain size of $16.7 \mu \mathrm{m}$ was provided by NASA. The composition (in wt.\%) of the LSHR alloy is: $20.7 \mathrm{Co}, 12.5 \mathrm{Cr}, 3.5 \mathrm{Ti}, 3.5 \mathrm{Al}$, $4.3 \mathrm{~W}, 2.7 \mathrm{Mo}, 1.6 \mathrm{Ti}, 1.5 \mathrm{Nb}, 1.5 \mathrm{Ta}, 0.05 \mathrm{Zr}, 0.03 \mathrm{C}, 0.03 \mathrm{~B}$, bal. Ni. Two fatigue tests were conducted on polished plain bend bar specimens $(4 \mathrm{~mm} \times 4 \mathrm{~mm} \times 53 \mathrm{~mm})$ under three-point loading on an Instron hydraulic testing machine in air at $650{ }^{\circ} \mathrm{C}$. A trapezoidal 1-1-1-1 loading waveform with a load ratio of 0.1 was employed for the fatigue tests. This incorporates a 1s loading ramp from minimum load up to maximum load, a $1 \mathrm{~s}$ dwell at maximum load, a 1s unloading ramp from maximum load down to minimum load, and a $1 \mathrm{~s}$ dwell at minimum load. The applied maximum load was $1500 \mathrm{~N}$, which is predicted to achieve a maximum strain of $0.8 \%$ at the top central surface of the specimen based on an elastic-plastic finite element simulation. One test was interrupted at regular intervals, and the specimen cooled and then examined under optical microscopy (OM) to observe crack evolution. The comparison test was run continuously to the same number of cycles to produce similar cracking levels as observed in the interrupted test.

A JEOL JSM 6500F field emission gun scanning electron microscopy (SEM) equipped with an Oxford Instruments Energy dispersive X-ray spectrometry (EDS) and INCA analysis system was employed to examine GB oxidation at an acceleration voltage of $15 \mathrm{kV}$. EDS analysis of composition across GBs was conducted to reveal the degree of GB oxidation and to assess possible oxides formed. This EDS measurement was based on the library standards and quantification algorithms employed in the Oxford Inca software system. The interval between two EDS analysis points was $1 \mu \mathrm{m}$. Electron backscatter diffraction (EBSD) was conducted using HKL Channel 5 software provided by Oxford Instruments to reveal the dependence of GB oxidation on grain orientation. A step size of $1 \mu \mathrm{m}$ and a grain boundary tolerance angle of $2^{\circ}$ were employed for EBSD mapping. After EBSD indexing, the three 
Euler angles $\left(\varphi_{1}, \varnothing, \varphi_{2}\right)$ of the grains of interest were extracted to determine the rotation matrix $g$ which connects the sample coordinates and crystal coordinates using following equation [23]:

$\boldsymbol{g}=\left[\begin{array}{ccc}\cos \varphi_{1} \cos \varphi_{2}-\sin \varphi_{1} \sin \varphi_{2} \cos \emptyset & \sin \varphi_{1} \cos \varphi_{2}+\cos \varphi_{1} \sin \varphi_{2} \cos \emptyset & \sin \varphi_{2} \sin \emptyset \\ -\cos \varphi_{1} \sin \varphi_{2}-\sin \varphi_{1} \cos \varphi_{2} \cos \emptyset & -\sin \varphi_{1} \sin \varphi_{2}+\cos \varphi_{1} \cos \varphi_{2} \cos \emptyset & \cos \varphi_{2} \sin \emptyset \\ \sin \varphi 1 \sin \emptyset & -\cos \varphi 1 \sin \emptyset & \cos \emptyset\end{array}\right]$

By converting the loading direction from the sample coordinates to crystal coordinates using the rotation matrix and the following equation [23],

$L_{\text {crystal }}=\boldsymbol{g} L_{\text {sample }}(2)$,

where $L$ is a vector that represents the loading direction,

it is possible to determine the Schmid factor of the 12 primary slip systems (i.e. $\{111\}<110>$ ) in each grain of interest [23].

\section{Results}

Fig. 1 shows the crack evolution in the region (top central surface) which experiences maximum elastic stress in the interrupted test. It was found that cracks preferentially initiated at the GBs adjacent to the edge of the specimen due to stress concentration at this region, although the very early stages of crack initiation were not captured. After crack initiation, no substantial crack propagation phase was observed during the majority of the fatigue life, but discrete GB oxide cracking can be discerned as indicated by the arrows. On further cycling of the specimen, significant crack coalescence occurred along the oxidised GBs within $\sim 100$ cycles of the end of fatigue life. As shown in the SEM image, the crack paths are predominantly intergranular, and bulged oxides can be seen at GBs adjacent to the main crack path. Similar oxides can also be found along the crack path, although they are much less evident, which is probably associated with oxide fragmentation during cracking.

More detailed examination of GB oxides in the interrupted test is presented in Fig.2. Figs.2 (a) and (b) show most of the oxides on the top central surface form at GBs inclined normal to the applied tensile stress (which may be expected to experience the maximum opening stress) although a few oxides were also observed to form at GBs inclined parallel to the tensile stress direction. In addition, occasional slip band oxidation and cracking can be observed within certain grains. A close-up of the GB oxides is shown in the inset image in Fig.2 (a), which 
shows the crystallographic morphology of the bulged GB oxides as well as oxide particles within grains. It is interesting to note that not all the GBs inclined normal to the tensile stress direction are decorated with bulged oxides, indicating that GB character (or grain orientation) may also influence oxide formation. Bulged GB oxide cracking is indicated by the arrows as shown in Fig.2 (b) along with a close-up shown in the inset image showing how cracks propagate along GBs with bulged oxides. The bulged GB oxide ahead of a crack tip appears to be intact, indicating that the oxide formation occurs prior to crack initiation and crack initiation itself occurs via bulged oxide cracking. Moreover, it is found that some GBs are partly decorated with bulged oxides. Ahead of the bulged oxides, the GB seems to be decorated with much thinner/flatter oxides, which indicates a possible transition from these thin/relatively flat oxides to the wide/bulged oxides.

1625 cycles 2550 cycles 2675 cycles 2800 cycles 2925 cycles 3025 cycles 3025 cycles

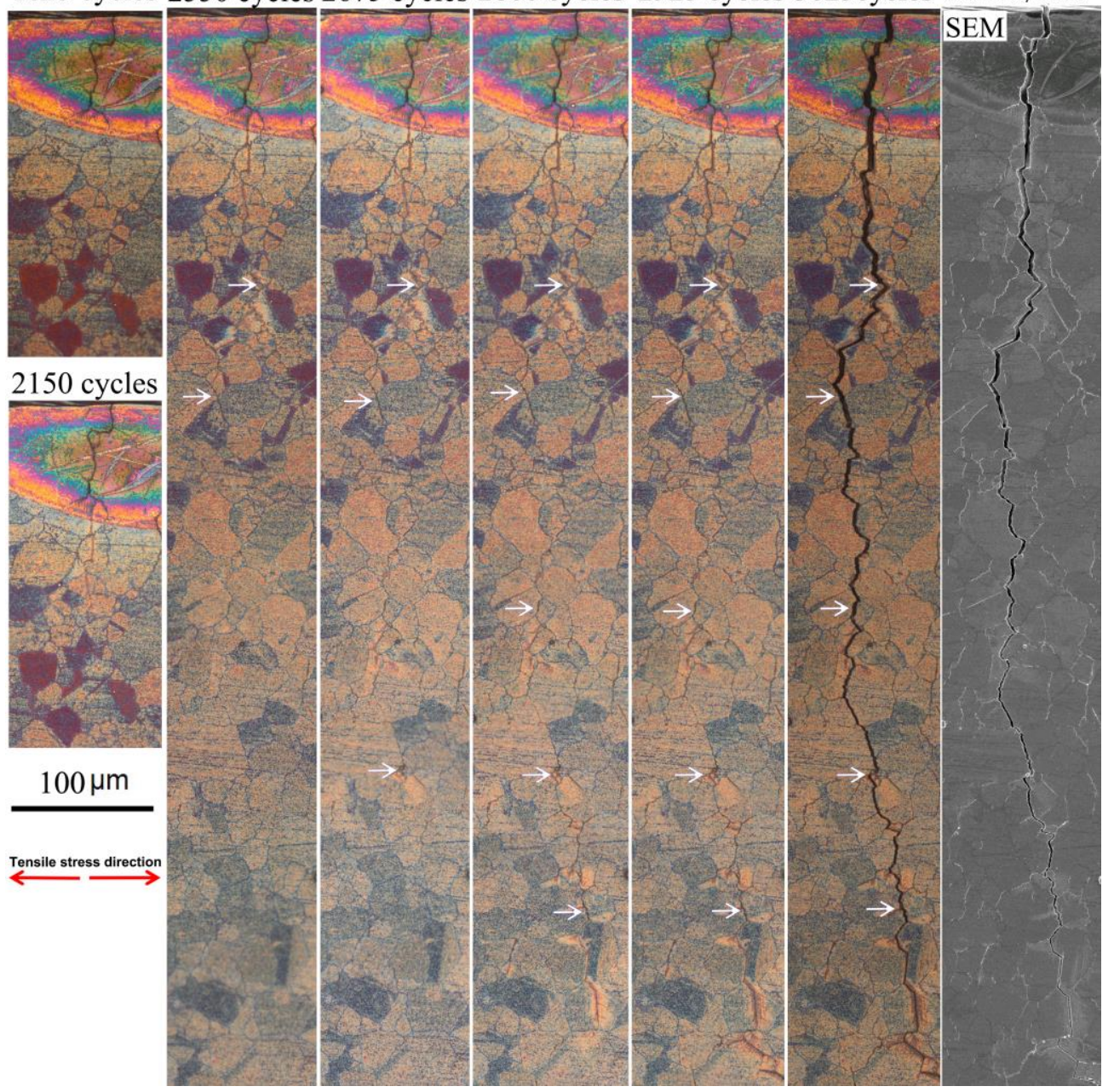

Fig.1 Crack field evolution at the top central surface in the interrupted test at $650^{\circ} \mathrm{C}$ observed under OM and SEM. The colouration within the grains is caused by oxidation. The arrows indicate discrete GB oxide cracking. 

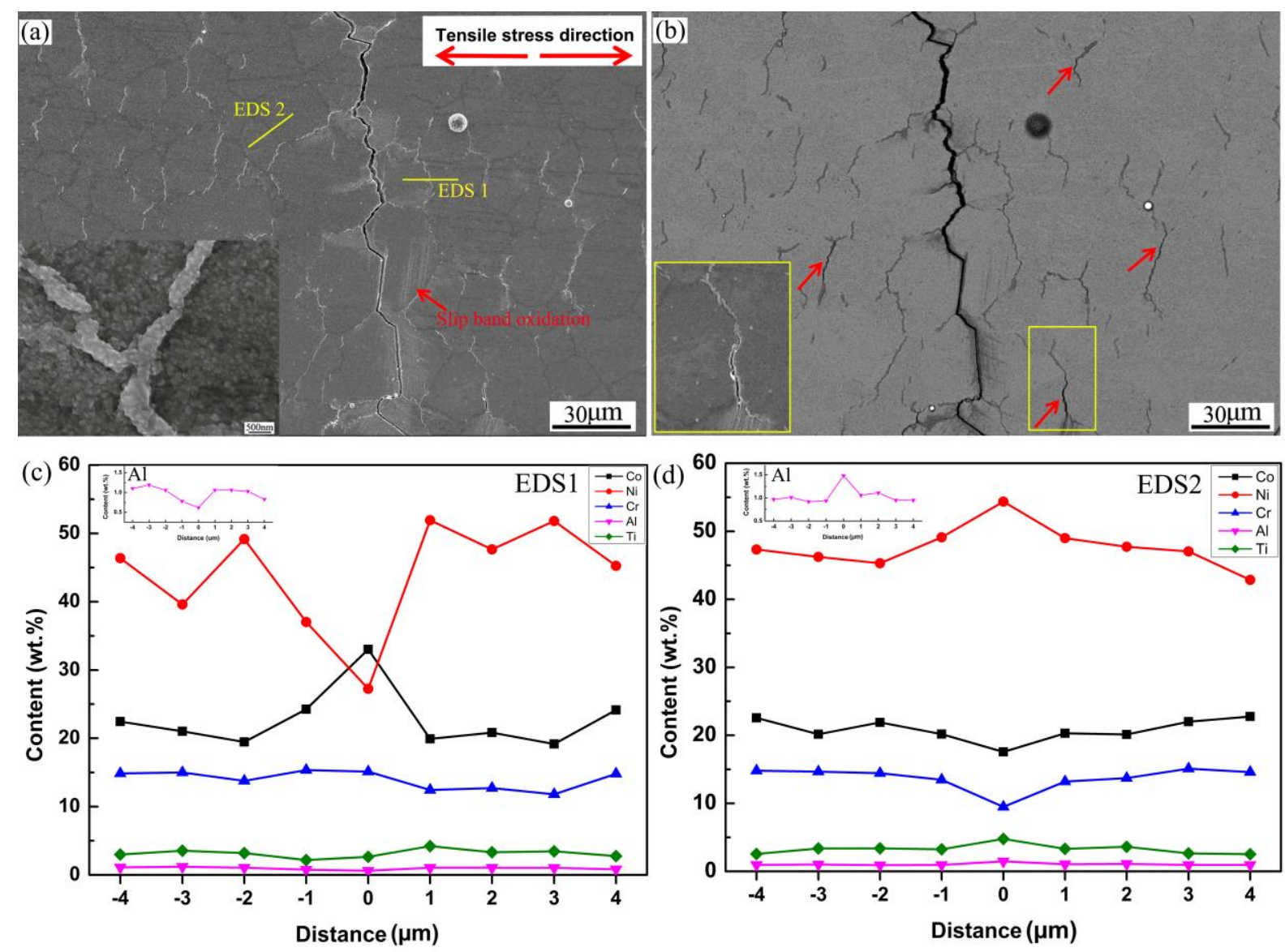

Fig. 2 (a) secondary electron image and (b) backscatter electron image of GB oxidation and cracking in the LSHR alloy; compositional profile across grain boundary (c) with bulged oxides and (d) without bulged oxides from EDS analysis. EDS analysis regions are highlighted by yellow lines shown in (a). A close-up of GB bulged oxides is inserted in (a) and a close-up of GB oxide cracking is inserted in (b). The compositional profiles of Al with a rescaled $y$ axis are inserted into (c) and (d) respectively.

Fig. 2 (c) shows the concentration variation of $\mathrm{Ni}, \mathrm{Co}, \mathrm{Cr}$, $\mathrm{Ti}$ and $\mathrm{Al}$ tracking across a $\mathrm{GB}$ with a bulged oxide analysed by EDS. "0" in the graph indicates the location of the GB. Significant enrichment of $\mathrm{Co}$ and slight enrichment of $\mathrm{Cr}$, along with a depletion of other metallic elements exist at the GB region, indicating that the bulged GB oxide is mainly a Corich oxide complex. Fig.2 (d) presents the compositional profile across a GB with a thin/flat oxide. A depletion of $\mathrm{Co}$ and $\mathrm{Cr}$ is observed, whilst an enrichment of $\mathrm{Ni}$, Ti and $\mathrm{Al}$ at the GB region is identified, although the extent of this enrichment is not as significant as that of Co at the GB with a bulged oxide. This qualitative variation of the GB compositional profile indicates that the GBs without bulged Co-rich oxide complex are decorated with a Ni/Ti/Alrich oxide complex. Additionally, it is found that the content of $\mathrm{Al}$ is lower than its original 
content either at GBs or within grains and the content of $\mathrm{Cr}$ is relatively higher than its original content within grains, this is believed to be associated with the formation of an internal alumina layer and an outer layer of chromium oxide as observed in Ni-based superalloys [12, 18, 22, 27-29]. However, it should be noted that point out that the elements in the regions nearby and underneath the GB oxides may also contribute to the composition of the GB oxides due to the relatively large electron beam size used for EDS analysis and the large activation volume. The depletion of $\mathrm{Ni}$ at a GB with bulged oxides may not be able to rule out the existence of GB $\mathrm{Ni}$ oxides as the enrichment of $\mathrm{Co}$ in a constant activation volume usually corresponds to a depletion of the matrix element (i.e. Ni). Moreover, the Ni oxide and Co oxide have the same crystal structure and similar lattice parameter, and they are apt to form a single-phase solid solution oxide scale $[18,30]$. Similarly, the depletion of Co and $\mathrm{Cr}$ in the $\mathrm{Ni} / \mathrm{Al} / \mathrm{Ti}$-rich oxide complex may not be able to rule out the existence of Co and $\mathrm{Cr}$ oxides in the thin/flat GB oxides. In this paper therefore the bulged Co-rich oxide complex and the thin/flat $\mathrm{Ni} / \mathrm{Ti} / \mathrm{Al}$-rich oxide complex are purely phenomenological definition based on the morphology and the "apparent" composition of GB oxides.

Fig.3 illustrates the dependence of the GB oxidation and cracking on grain orientation in the LSHR alloy. The region containing crack paths and GB oxides on the top central surface was chosen for EBSD mapping as shown in Fig.3 (a). The grain orientation of the chosen area is shown in Fig.3 (b), exhibiting many twins. The Schmid Factor (SF) map was derived from the grain orientation map by considering the direction of applied tensile stress as parallel to the length direction of the specimen. As shown in Fig.3 (c), crack paths appear to preferentially locate at the boundaries of high SF grain/low SF grain. Similarly, it is found the bulged oxides also tend to form at high SF grain/low SF grain boundaries as indicated by the arrows in Fig. 3 (a) and (c).

Figs. 3(d) and (e) shows the maximum SF of the grains neighbouring bulged GB oxides in the regions highlighted in Fig. 3(a). It can be found that the formation of bulged GB oxides is associated with the large difference in SF between grains (as indicated by the arrows), rather than simply a high SF value, as bulged GB oxides are also observed at the boundary of grains with a low SF. The dependence of bulged GB oxide formation on the difference of the SF may indicate that the oxidation process is related to the strain localisation, because dislocations are expected to pile up at boundaries of grains with a large difference in SF as a result of the hindrance of slip transmission (plastic and elastic incompatibility) at these regions $[23,31]$. The higher dislocation density associated with higher local strain can 
provide short-circuit paths for the diffusion of oxide-forming elements, and thereby it accelerates the oxide formation. In addition, the formation of oxides within slip bands shown in Fig.2 (a) is further evidence that the formation of oxides is indeed related to the local strain.
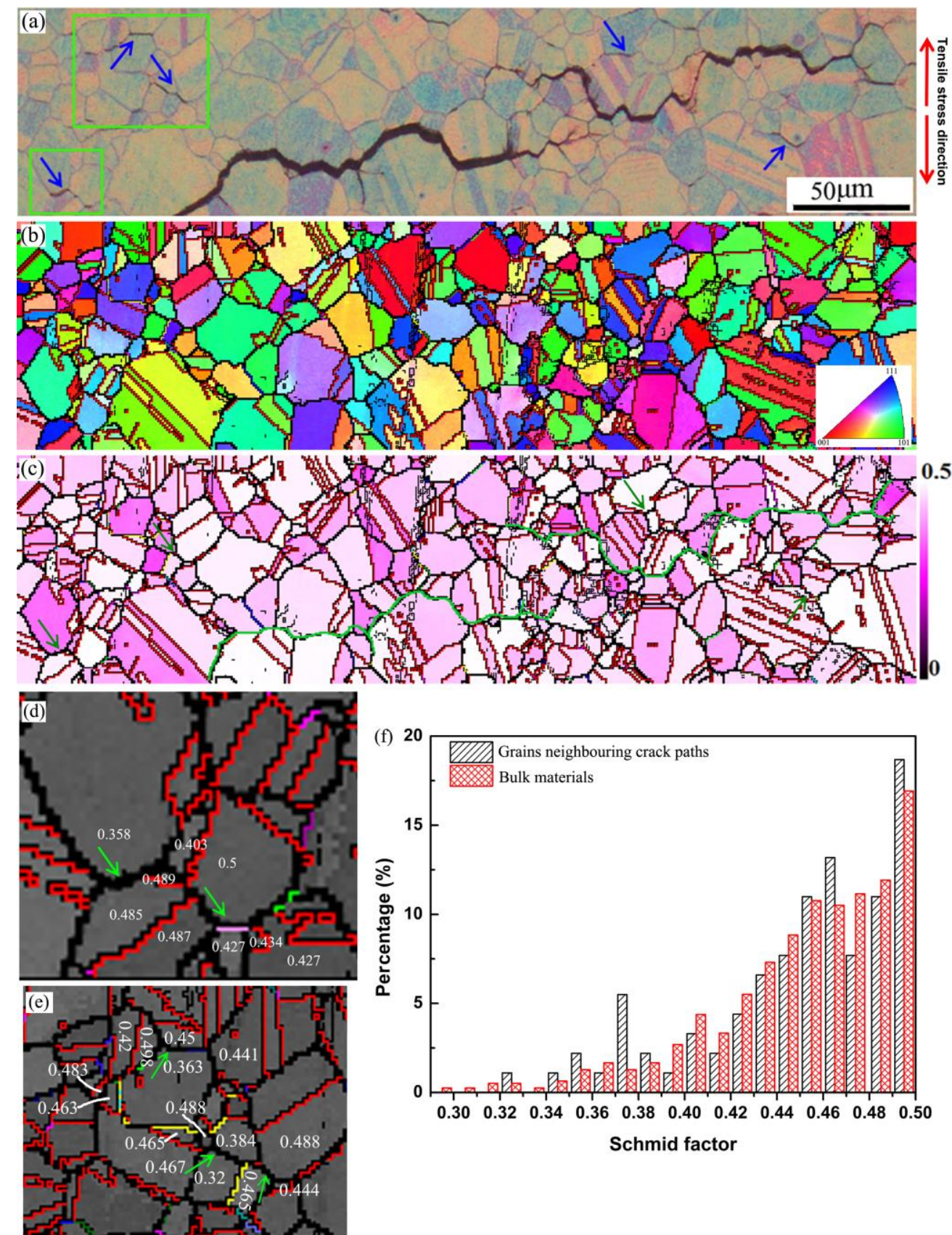

Fig.3 (a) Morphology of GB oxidation and cracking in the LSHR alloy observed under OM; (b) grain orientation map; (c) colour-coded SF map of the region shown in (a), crack paths are outlined on the SF map by green lines; (d) and (e) maximum SF of the grains of interest highlighted at the bottom and top shown in (a) respectively, the GBs with bulged oxides are indicated by the green arrows; and (f) SF distribution of grains neighbouring the crack paths and bulk materials. 
The distribution of the maximum SF of grains neighbouring the crack paths and $\sim 800$ grains in the bulk material are presented in Fig. 3 (f). It shows that most of the grains in the bulk material have slip systems associated with the relatively high SF $(>0.43)$ under the applied load conditions, and takes on a unimodal distribution. Conversely, a weak bimodal distribution of the SF of grains neighbouring the crack paths is observed, which is probably related to the fact that the cracked grain boundaries are associated with grains of differing SF. The weak trend of the bimodal distribution indicates that the GB oxidation and cracking may not necessarily require a very large difference in SF between neighbouring grains.

\section{Discussion}

As shown in the EBSD analysis, the formation of bulged Co-rich GB oxide complex and GB cracking are closely associated with grain orientation, although it might be argued that the formation of the bulged GB oxide complex could be related to GB cracking and subsequent oxygen ingress into the cracked GBs. However, the continuous observation of crack evolution and the observed intact bulged oxide complex ahead of the crack tip on the specimen surface (shown in Figs. 1 and 2) indicate that the formation of a bulged oxide complex occurs prior to GB cracking. In addition, the observed layered oxide intrusion ahead of a propagating crack tip in a similar disc alloy RR1000 and the intact oxide tip along with cracked oxide wake which was found at a secondary crack tip in the LSHR alloy (by metallographic sectioning the fracture surface after long fatigue crack growth test) further verify that GB oxidation occurs prior to GB cracking $[7,18]$.

In fact, the difference in the morphology and "apparent" composition between the bulged Co-rich oxide complex and thin/flat $\mathrm{Ni} / \mathrm{Ti} / \mathrm{Al}$ oxide complex at GBs can be rationalised by the difference in the accumulated local plastic strain brought about by grain misorientation. According to studies conducted on RR1000 by Foss et al. [28] and Karabela et al. [20], the applied load does not influence the oxidation products, but enhances the oxidation processes. Thus, the difference in the morphology and "apparent" composition between bulged Co-rich oxide complex and thin/flat $\mathrm{Ni} / \mathrm{Ti} / \mathrm{Al}$ oxide complex at GBs may only reflect the difference in the extent of GB oxidation rather than the difference in the nature of GB oxides. Although the analysis of the composition of GB oxides conducted in this study is preliminary and the structure of these GB oxides needs further investigation, it is reasonable to assume that layered oxides form, i.e. thermodynamic unstable oxides such as $\mathrm{CoO}$ and $\mathrm{NiO}$ at the surface and thermodynamic stable oxides such as $\mathrm{Cr}_{2} \mathrm{O}_{3}, \mathrm{TiO}_{2}$ and $\mathrm{Al}_{2} \mathrm{O}_{3}$ at the subsurface. Such 
layered oxides are usually observed in stressed and unstressed disc alloys RR1000 and ME3 $[12,18,20,28]$, so may also form in the LSHR alloy due to the similarity in alloying composition and the comparable/equivalent test conditions. At the beginning of the fatigue loading process at elevated temperature, non-selective oxidation can occur at the specimen surface, and thereby $\mathrm{Ni}$ and $\mathrm{Co}$ oxidise rapidly and form a continuous single-phase solid solution scale due to their abundance in the alloy and the same crystal structure of $\mathrm{NiO}$ and $\mathrm{CoO}[18,30]$. Meanwhile, strain accumulates at boundaries between high and low SF grains [31]. Due to the formation of the surface $\mathrm{Ni} / \mathrm{Co}$ oxide layer, internal oxidation (i.e. selective oxidation of $\mathrm{Cr}$, $\mathrm{Ti}$ and $\mathrm{Al}$ ) starts to occur, particularly along GBs due to the relatively high density of dislocations and vacancies which are expected to accelerate the diffusion of oxideforming elements, as a consequence of the reduced oxygen partial pressure at the interface of surface oxide layer/alloy matrix. Since the strain accumulation is greatest at boundaries between high and low SF grains (which is in turn associated with high stored strain energy and high defect density), this further accelerates the internal oxidation process at these grain boundaries via enhancing inward diffusion of oxygen, producing an abundance of $\mathrm{Ni}$ and $\mathrm{Co}$ by consuming $\mathrm{Ti}$ and $\mathrm{Al}$ (which is accompanied by dissolution of $\gamma^{\prime}$ precipitates). With the inward diffusion of oxygen during internal oxidation, a counter-current transport of oxygen vacancies or other metallic elements (cations) is expected to occur [28, 32, 33]. Therefore, it is reasonable to infer that the abundant $\mathrm{Ni}$ and $\mathrm{Co}$ then migrate outward to form $\mathrm{Ni} / \mathrm{Co}$ oxides at surface GBs by short-circuit diffusion along these strain-accumulated grain boundaries, resulting in the apparent bulged height at surface GBs. For those GBs with less strain accumulation, the relatively thin/flat oxide complex therefore forms.

On the basis of the present findings, it seems that SAGBO is the most likely mechanism for oxidation enhanced short fatigue cracking in the LSHR alloy. Compared with the studies of isothermal oxidation/cyclic oxidation for the disc alloys, the oxidation under (cyclic) stress exhibits a greater discrepancy between grain boundary and grain interior [12, 22]. The grain boundary oxidation is much more significant than that in the grain interior. The main oxide contributing to the oxidation enhanced short crack cracking is believed to be a bulged GB Co-rich oxide complex. The formation of the bulged GB Co-rich oxide complex appears to be related to both the local accumulated strain at GBs and grain misorientation. Although intergranular crack initiation and propagation are commonly observed in turbine disc alloys due to oxidation (e.g. intergranular crack initiation and propagation in ME3 due to formation of $\mathrm{Cr}_{2} \mathrm{O}_{3} / \mathrm{Al}_{2} \mathrm{O}_{3} / \mathrm{TiO}_{2}$ and the $\mathrm{Ni}$ oxides in In 718) $[3,16]$, the fatigue cracking associated with 
formation of apparent Co-rich oxide complex is much less common, which is probably related to the comparatively high content of Co in the LSHR alloy. In addition, studies that have been conducted to reveal the oxidation ahead of a crack tip or underneath the fracture surface in high temperature fatigue tests or during sustained load tests of turbine disc superalloys $[5,17,19,20]$ rarely show the existence of such apparent GB Co-rich oxides except for Kitaguchi's work on RR1000 [18]. The observed bulged GB Co-rich oxide complex and the cracking occurring in this kind of oxide complex suggest that the oxidation of Co under cyclic load at elevated temperatures may be an important issue in these newer disc alloys and may accelerate crack initiation and propagation, especially in high Cocontaining disc alloys, such as the LSHR alloy investigated in this study as well as RR1000 and TMW-4M3 [18, 25, 26].

\section{Conclusion}

In summary, following conclusions can be made based on the aforementioned results and discussion:

1. Plentiful crack initiation mainly occurs at bulged GB Co-rich oxide complexes at $650^{\circ} \mathrm{C}$ in the LSHR alloy due to oxide cracking. Cracks subsequently propagate along oxidised GBs and exhibit significant crack coalescence at the final stages of fatigue life.

2. Formation of bulged GB Co-rich oxide complexes is closely related to the strain localisation which is associated with grain orientation and applied stress. The boundaries of high SF grain/low SF grain are preferential site for formation of bulged Co-rich oxide complexes.

3. A thin/flat $\mathrm{Ni} / \mathrm{Ti} / \mathrm{Al}$-rich oxide complex is also found to form at GBs, but it appears to be much less detrimental for fatigue crack initiation and propagation.

\section{Acknowledgement}

Thanks are due to the EPSRC (Grant EP/K027271/1) and China Scholarship Council for funding support, and to NASA for the supply of the LSHR alloys.

\section{References}

[1] S. Everitt, R. Jiang, N. Gao, M. J. Starink, J. W. Brooks, P. A. S. Reed (2013) Comparison of fatigue crack propagation behaviour in two gas turbine disc alloys under creep-fatigue conditions: 
evaluating microstructure, environment and temperature effects, Materials Science and Technology 29: 781-787.

[2] H.T. Pang, P.A.S. Reed (2007) Microstructure effects on high temperature fatigue crack initiation and short crack growth in turbine disc nickel-base superalloy Udimet $720 \mathrm{Li}$, Materials Science and Engineering: A 448: 67-79.

[3] R. Molins, G. Hochstetter, J.C. Chassaigne, E. Andrieu (1997) Oxidation effects on the fatigue crack growth behaviour of alloy 718 at high temperature, Acta Materialia 45: 663-674.

[4] J. Tong, J. Byrne (1999) Effects of frequency on fatigue crack growth at elevated temperature, Fatigue \& Fracture of Engineering Materials \& Structures 22: 185-193.

[5] L. Ma, K.-M. Chang (2003) Identification of SAGBO-induced damage zone ahead of crack tip to characterize sustained loading crack growth in alloy 783, Scripta Materialia 48: 1271-1276.

[6] T.P. Gabb, J. Gayda, J. Telesman, L.J. Ghosn, A. Garg (2013) Factors influencing dwell fatigue life in notches of a powder metallurgy superalloy, International Journal of Fatigue 48: 55-67.

[7] R. Jiang, S. Everitt, M. Lewandowski, N. Gao, P.A.S. Reed (2014) Grain size effects in a Ni-based turbine disc alloy in the time and cycle dependent crack growth regimes, International Journal of Fatigue 62: 217-227.

[8] S. Dalby, J. Tong (2005) Crack growth in a new nickel-based superalloy at elevated temperature: part I - effect of loading waveform and frequency on crack grwoth, Journal of Materials Science 40: $1217-1228$.

[9] J. Tong, S. Dalby, J. Byrne (2005) Crack growth in a new nickel-based superalloy at elevated temperature: part III - characterisation, Journal of Materials Science 40: 1237-1243.

[10] R. Jiang, S. Everitt, N. Gao, K. Soady, J. W Brooks, P. A. S. Reed (2015) Influence of oxidation on fatigue crack initiation and propagation in turbine disc alloy N18, International Journal of Fatigue 75: 89-99.

[11] T. Connolley, P.A.S. Reed, M.J. Starink (2003) Short crack initiation and growth at $600^{\circ} \mathrm{C}$ in notched specimens of Inconel718, Materials Science and Engineering: A 340: 139-154.

[12] C. K. Sudbrack, S. L. Draper, T. T. Gorman, J. Telesman, T. P. Gabb, D. R. Hull. Oxidation and the Effects of High Temperature Exposures on Notched Fatigue Life of an Advanced Powder Metallurgy Disk Superalloy. In: Eric S. Huron, Roger C. Reed, Mark C. Hardy, Michael J. Mills, Rick E. Montero, Pedro D. Portella, Jack Telesman, editors. TMS Superalloys 2012, pp. 853-862.

[13] U. Krupp, W.M. Kane, C. Laird, C.J. McMahon (2004) Brittle intergranular fracture of a Ni-base superalloy at high temperatures by dynamic embrittlement, Materials Science and Engineering: A 387: 409-413.

[14] J.A. Pfaendtner, C.J. McMahon Jr (2001) Oxygen-induced intergranular cracking of a Ni-base alloy at elevated temperatures - an example of dynamic embrittlement, Acta Materialia 49: 33693377 .

[15] U. Krupp (2008) Improving the resistance to intergranular cracking and corrosion at elevated temperatures by grain-boundary-engineering-type processing, Journal of Materials Science 43: 39083916.

[16] E. Andrieu, R. Molins, H. Ghonem, A. Pineau (1992) Intergranular crack tip oxidation mechanism in a nickel-based superalloy, Materials Science and Engineering: A 154: 21-28.

[17] C.F. Miller, G.W. Simmons, R.P. Wei (2003) Evidence for internal oxidation during oxygen enhanced crack growth in P/M Ni-based superalloys, Scripta Materialia 48: 103-108.

[18] H.S. Kitaguchi, H.Y. Li, H.E. Evans, R.G. Ding, I.P. Jones, G. Baxter, P. Bowen (2013) Oxidation ahead of a crack tip in an advanced Ni-based superalloy, Acta Materialia 61: 1968-1981.

[19] C.F. Miller, G.W. Simmons, R.P. Wei (2001) Mechanism for oxygen enhanced crack growth in inconel 718, Scripta Materialia 44: 2405-2410.

[20] A. Karabela, L.G. Zhao, J. Tong, N.J. Simms, J.R. Nicholls, M.C. Hardy (2011) Effects of cyclic stress and temperature on oxidation damage of a nickel-based superalloy, Materials Science and Engineering: A 528: 6194-6202.

[21] L. Viskari, M. Hörnqvist, K. L. Moore, Y. Cao, K. Stiller (2013) Intergranular crack tip oxidation in a Ni-base superalloy, Acta Materialia 61:3630-3639.

[22] J.H. Chen, P.M. Rogers, J.A. Little (1997) Oxidation behavior of several chromia-forming commercial nickel-base superalloys, Oxidation of Metals 47: 381-410. 
[23] W.Z. Abuzaid, M.D. Sangid, J.D. Carroll, H. Sehitoglu, J. Lambros (2012) Slip transfer and plastic strain accumulation across grain boundaries in Hastelloy X, Journal of the Mechanics and Physics of Solids 60: 1201-1220.

[24] J. Gayda, T. P. Gabb, P. T. Kantzos. The effect of dual microstructure heat treatment on an advanced Ni-based disk alloy. In: Green KA, Harada H, Howson TE, Pollock TM, Reed RC, Schirra JJ, et al., editors. TMS superalloy 2004. Warrendale (PA): The Minerals, Metals \& Materials Society; 2004. p. 323-340.

[25] T. P. Gabb, J. Gayda, J. Telesman. Thermal and mechanical property characterization of the advanced disk alloy LSHR, NASA report NASA/TM-2005-213645; 2005.

[26] Y. Yuan, Y.F. Gu, T. Osada, Z.H. Zhong, T. Yokokawa, H. Harada (2012) Deformation mechanisms in a new disc superalloy at low and intermediate temperatures, Scripta Materialia 67: 137-140.

[27] H.S. Kitaguchi, M.P. Moody, H.Y. Li, H.E. Evans, M.C. Hardy, S. Lozano-Perez (2015) An atom probe tomography study of the oxide-metal interface of an oxide intrusion ahead of a crack in a polycrystalline Ni-based superalloy, Scripta Materialia 97(0): 41-44.

[28] B.J. Foss, M.C. Hardy, D.J. Child, D.S. McPhail, B.A. Shollock (2014) Oxidation of a Commercial Nickel-Based Superalloy under Static Loading, JOM 66(12): 2516-2524.

[29] A. Sato, Y.L. Chiu, R.C. Reed (2011) Oxidation of nickel-based single-crystal superalloys for industrial gas turbine applications, Acta Materialia 59(1): 225-240.

[30] N. Birks, G. H. Meier, F. S. Pettit (2009) Introduction to the High Temperature Oxidation of Metals, $2^{\text {nd }}$ edn., Cambridge University Press.

[31] Z.J. Zhang, P. Zhang, L.L. Li, Z.F. Zhang (2012) Fatigue cracking at twin boundaries: Effects of crystallographic orientation and stacking fault energy, Acta Materialia 60: 3113-3127.

[32] S. C. Tsai, A.M. Huntz, C. Dolin (1996) Growth mechanism of $\mathrm{Cr}_{2} \mathrm{O}_{3}$ scales: oxygen and chromium diffusion, oxidation kinetics and effect of yttrium, Materials Science and Engineering: A 212: 6-13.

[33] H.E. Evans, H.Y. Li, P. Bowen (2013) A mechanism for stress-aided grain boundary oxidation ahead of cracks, Scripta Materialia 69: 179-182. 